\title{
DTM generation from aerial photo and Worldview-3 images by using different DSM filtering methods
}

\section{Catur Aries Rokhmana, Ahmad Ridho Sastra}

Catur Aries Rokhmana, Ahmad Ridho Sastra, "DTM generation from aerial photo and Worldview-3 images by using different DSM filtering methods," Proc. SPIE 11311, Sixth Geoinformation Science Symposium, 113110J (21 November 2019); doi: 10.1117/12.2547622

SPIE Event: Sixth Geoinformation Science Symposium, 2019, Yogyakarta, Indonesia 


\title{
DTM GENERATION FROM AERIAL PHOTO AND WORLDVIEW-3 IMAGES BY USING DIFFERENT DSM FILTERING METHOD
}

\author{
Catur Aries Rokhmana ${ }^{1}$, Ahmad Ridho Sastra ${ }^{2}$ \\ ${ }^{1}$ Departmenet of Geodetic Engineering Gadjah Mada University, Jl. Grafika No. 2 Yogyakarta, \\ Indonesia, caris@ugm.ac.id \\ ${ }^{2}$ Departmenet of Geodetic Engineering Gadjah Mada University, Jl. Grafika No. 2 Yogyakarta, \\ Indonesia, sastraridho22@gmail.com
}

\begin{abstract}
The goal of this study is to analyze the suitability of the filtering method towards the generation of Digital Terrain Model (DTM) from the Digital Surface Model (DSM). In this case, DSM was produced by optical images sensor with stereoscopic viewing. Two kinds of optical images that used which are Very High-Resolution Satellite (VHRS) image Worldview-3 and Aerial Photograph from Drone mission. Furthermore, there are three different DSM filtering methods are used which are Elevation Threshold with Expand Window (ETEW), 2D Morphological Square (Morph 2D Filter), and Adaptive TIN (A-Tin). The ALDPAT v.1.0 software was used to implement that algorithm. Also, the visual interpretation from their correspondent images is used to evaluate the quality of the filtering process. A good filtering result will remove the point that not represent a terrain. The missing of the non-ground point due to the filtering process than filling by implementation the same interpolation algorithm. The ETEW and A-Tin filter algorithm are suitable for residential areas. While the A-Tin filter algorithm gives the best results in the vegetation area. An iterative filtering process should be implemented to make a non-terrain point completely remove. Further, an interpolation process should be made for filling the missing non-terrain point.
\end{abstract}

Keywords: DSM extraction, Aerial Photograph, World View 3, Filtering DSM, DTM

\section{INTRODUCTION}

Recently, the one map policy in Indonesia began to be encouraged for large-scale maps up to 1/10.000 - 1/5.000. Hence all efforts to encourage the acceleration of large-scale map production are always needed. Using the Very HighResolution Satellite (VHRS) imagery for mapping can be a good choice, due to its wide-area coverage. There are many kinds of researches that have used of VHRS imagery technique for producing a base map (see [1], [2], [3]). In general, The Base Map use DTM (Digital Terrain Model) as elevation information. Unfortunately, producing terrain point cloud from airborne or spaceborne with optical sensor camera is quite the challenge. The elevation point model extraction from spaceborne or aerial platform imagery only produces DSM (Digital Surface Model) instead of DTM. However, working with an aerial photo and VHRS image can produce a high spatial resolution that meets the need for large scale mapping. This study uses Worldview-3 stereo ready images that have a $30 \mathrm{~cm}$ x $30 \mathrm{~cm}$ spatial resolution. While the aerial photo uses 10cm Ground Sampling Distance (GSD). Furthermore, the DSM filtering process is one of the procedures that can be done for classifying terrain and non-terrain point cloud. The goal of this study is to analyze the suitability of the filtering methods towards the generation of Digital Terrain Model (DTM) from Digital Surface Model (DSM) extraction.

Some DSM filtering algorithms to classify between terrain and non-terrain point cloud has tried. There are three kind algorithm, which are: (1) Elevation Threshold with Expand Windows (ETEW), (2) 2D Morphological Square (Morph 2D Filter), and (3) Adaptive TIN (A-Tin) (see [4], [5], [6], [7]). The ALDPAT (Airborne LIDAR Data Processing and Analysis Tools) v.1.0 software was used to implement those algorithms. Although the ALDPAT program is designed for handling LIDAR data, it can be used for general point cloud data also. These filtering algorithms are implemented for residential and vegetation areas. The residential area mostly covered by houses objects. While vegetation area rather open area. The evaluation test has been made by visual interpretation to see the point remain. A good filtering result can remove a non-terrain point as much as possible. This visual interpretation can be done by using correspondence its orthoimage and orthophoto. Furthermore, an interpolation algorithm has to be done to fill the area missing point.

Sixth Geoinformation Science Symposium, edited by Sandy Budi Wibowo, Andi B. Rimba

Stuart Phinn, Ammar A. Aziz, Proc. of SPIE, Vol. 11311, 113110J · C 2019 SPIE

CCC code: $0277-786 \mathrm{X} / 19 / \$ 21 \cdot$ doi: $10.1117 / 12.2547622$ 


\section{IMAGE DATASHEET}

This study uses two kinds of landscape area which are (1) Mountainous area at The Tasikmalaya District and (2) SubUrban area at Probolinggo District. Table 1 shows the sample image of Orthoimage from Worldview-3 and Orthophoto from aerial photo. The DSM data from Worldview-3 has derived by stereo parallax with area-based image matching from stereo epipolar image. While the DSM from the aerial photo has derived by the parallax algorithm also.

Table 1. Image datasheet.

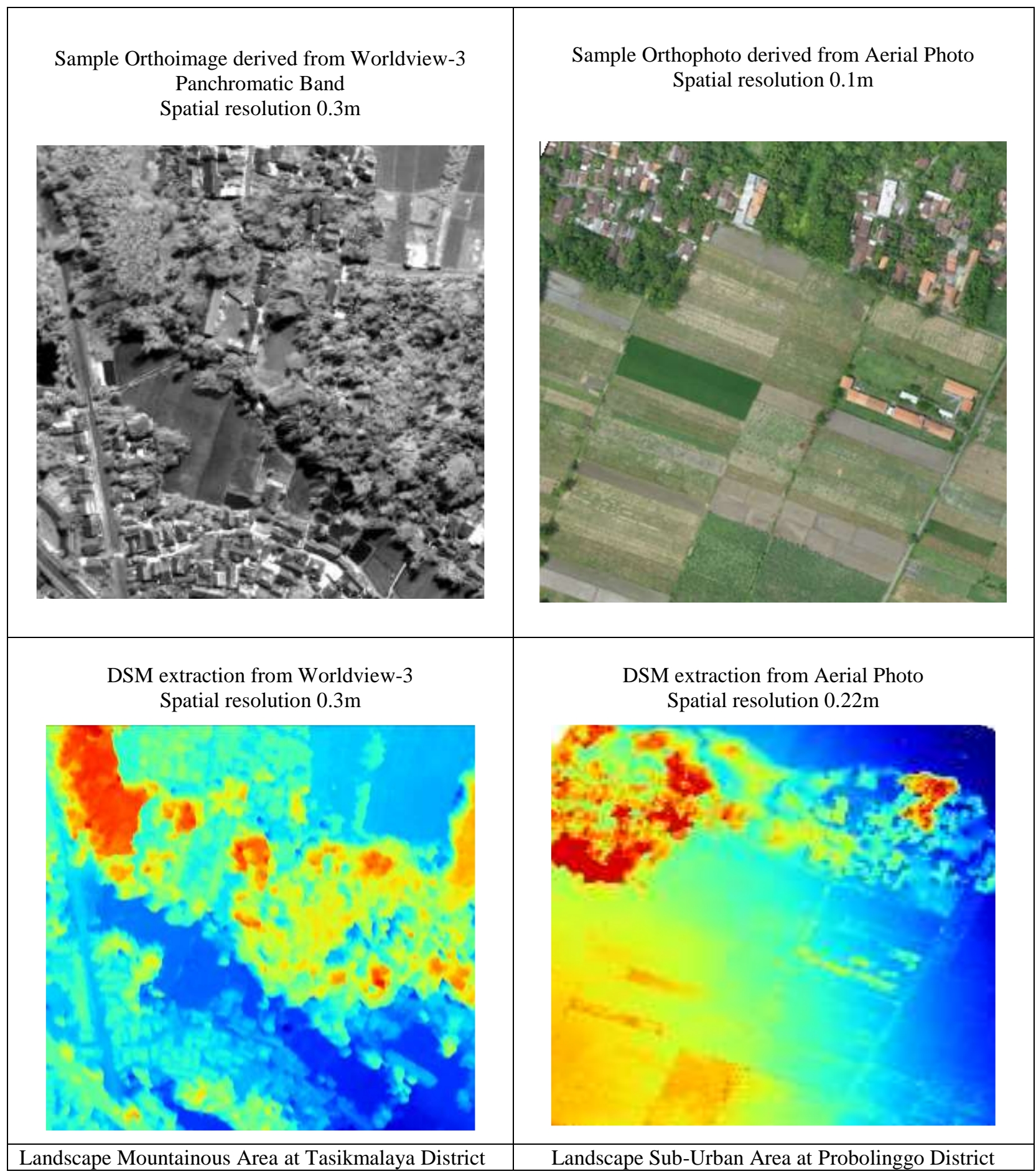




\section{METHODOLOGY}

The methodology is arranged to fulfil the research objectives effectively (see Figure 1). The most important steps are described as follows:

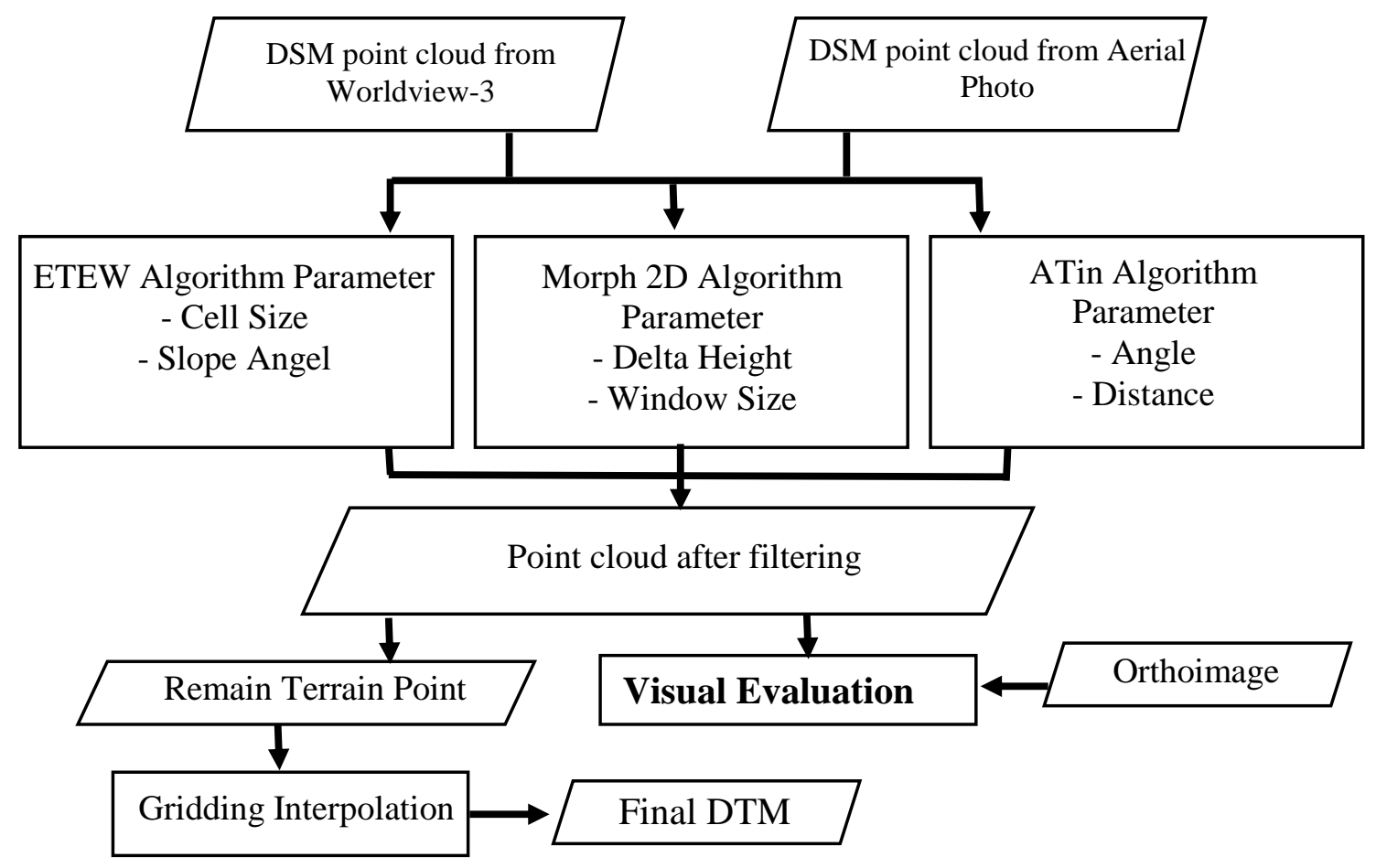

Figure 1. Workflow diagram of research

This research performs several phases of the procedure. First, acquisition of the DSM Point Cloud data from the WorldView-3 and DSM Point Cloud data from aerial photo data. Data is done filtering process using ALDPAT software in generating DTM. The DSM filtering process for both data uses the Method 3 algorithm which is ETEW, ATIN, and Morph 2D so that obtained 3 outputs from the DSM filtering process of the ALDPAT software. Once the cloud point is obtained from the DSM filtering process, the DTM resulting from the use of 3 algorithm methods, performed a visual evaluation of the data.

As for the concept explanation of the 3 algorithm methods used as follows:

a). ATEW is a filter method to identify and remove non-ground surface points such as buildings, trees, and vegetation. This filter method uses elevation that can be used to separate ground and non-ground. The ETEW Filter will divide the data set into an array of square cells and all points except for the minimum elevation to be discarded ( see [9])).

b). The Morph filter refers to a method to extract the features from the data to perform filtering by selecting the window size and setting the boundary of the elevation difference. This method will pay attention to the ground surface but does not pay attention to non-ground surfaces. The Morph filter is used to remove the non-ground point with the size of the window increasing, the removed point will also contain the ground point (see [4]).

c). The adaptive TIN which to select the ground point of the data set of aerial photographs and imagery using the distance at a point on the TIN surface. Adaptive TIN is one of the filters that can use the lowest point to depict ground level. The point is then triangulated to produce TIN (see [8]). 
(1) The three option Filtering algorithm are as follow:

ETEW: Elevation Threshold with Expand Windows (see [5], [7])

ETEW Filket Parmeters:
\begin{tabular}{|l|l|l|}
\hline Paremster & Volve & Tipe \\
\hline Width & 1.000000 & Double \\
Height & 1.000000 & Double \\
Max Z & 9999000000 & Double \\
Min Z & .9999 .000000 & Double \\
Slope & 1.000000 & Double \\
Loop Times & 10 & Irteger \\
\end{tabular}

Width: cell width

Height: cell height

Max Z: maximum elevation allowed for all ground points

Min Z: minimum elevation allowed for all ground points

Slope: slope factor in determining the threshold value of elevation differences

Loop Times: maximum number of iteration performed by the filter

ATin: Adaptive TIN (see [6], [7])

\begin{tabular}{|c|c|c|}
\hline Patameter & Volve & Type \\
\hline Coll Size & 1.000000 & Double \\
\hline$Z$ Difference & 0.200000 & Double \\
\hline Angle Theoshold & 0000000 & Doublo \\
\hline Init TrGird Size & 100.000000 & Double \\
\hline TileXWdth & 200000000 & Double \\
\hline Tile Y Height & 200.000000 & Double \\
\hline Tile Buffer & 20000000 & Double \\
\hline
\end{tabular}

Cell Size: cell size of a grid to parse the raw data.

$\mathrm{Z}$ difference: the threshold used to compare the distance from a point to a point beneath the TIN surface.

Angle Threshold: the default value is 0 where no angle threshold is used.

Init TriGrid Size: the initial size of a grid used to select seed points for the ground data set. A point with a minimum elevation within a grid cell is selected as a seed point.

Tile X Width: the width of a rectangle for applying ATIN filter. Delauney triangulation of LIDAR points for a large area is time consuming.

Tile Y Height: the height of rectangle for applying ATIN filter.

Tile Buffer: buffer size for a rectangle.

Morph 2D Filter: 2D Morphological Square (see [4], [7])

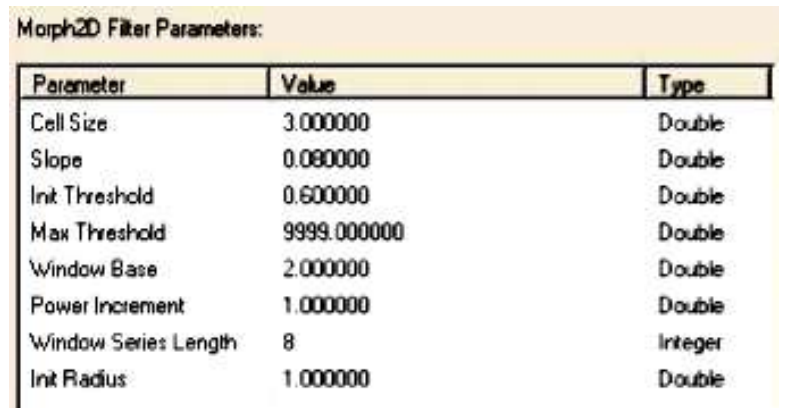

Cell size: cell size.

Slope: slope value used to determine the elevation difference threshold.

Init Threshold: the initial elevation difference threshold which approximates the error of LIDAR measurements (0.15$0.3 \mathrm{~m})$.

Max Threshold: the maximum elevation difference is an optional threshold which is set to a fixed height (e.g., the lowest building height) to ensure that building complexes in urban areas are removed

Window Base: base to determine the window size.

Power Increment: increment for computing a window series. Window Series Length: length of a window series.

Init Radius: initial search radius for nearest neighbor interpolation

Wind Series: window size series. Window size is computed by a power function: (window base)(window step). window size and slope value.

(2) The visual interpretation has done by using its correspondent orthophoto. A terrain point cloud should be remain, while the non-terrain point should be removed. Plotting remain point cloud at its orthophoto can be used to evaluate the filtering quality. 


\section{RESULT AND DISCUSSION}

\subsection{DSM Filtering}

Point cloud data from DSM filtering results with ALDPAT program can be seen in Table 2. and Table. 3The filtering results from the three algorithms produce different point removing in the same area. Experiments in residential and vegetation areas provide different responses by using difference filtering algorithms.

Table 2. Result from DSM Filtering in Residential Area

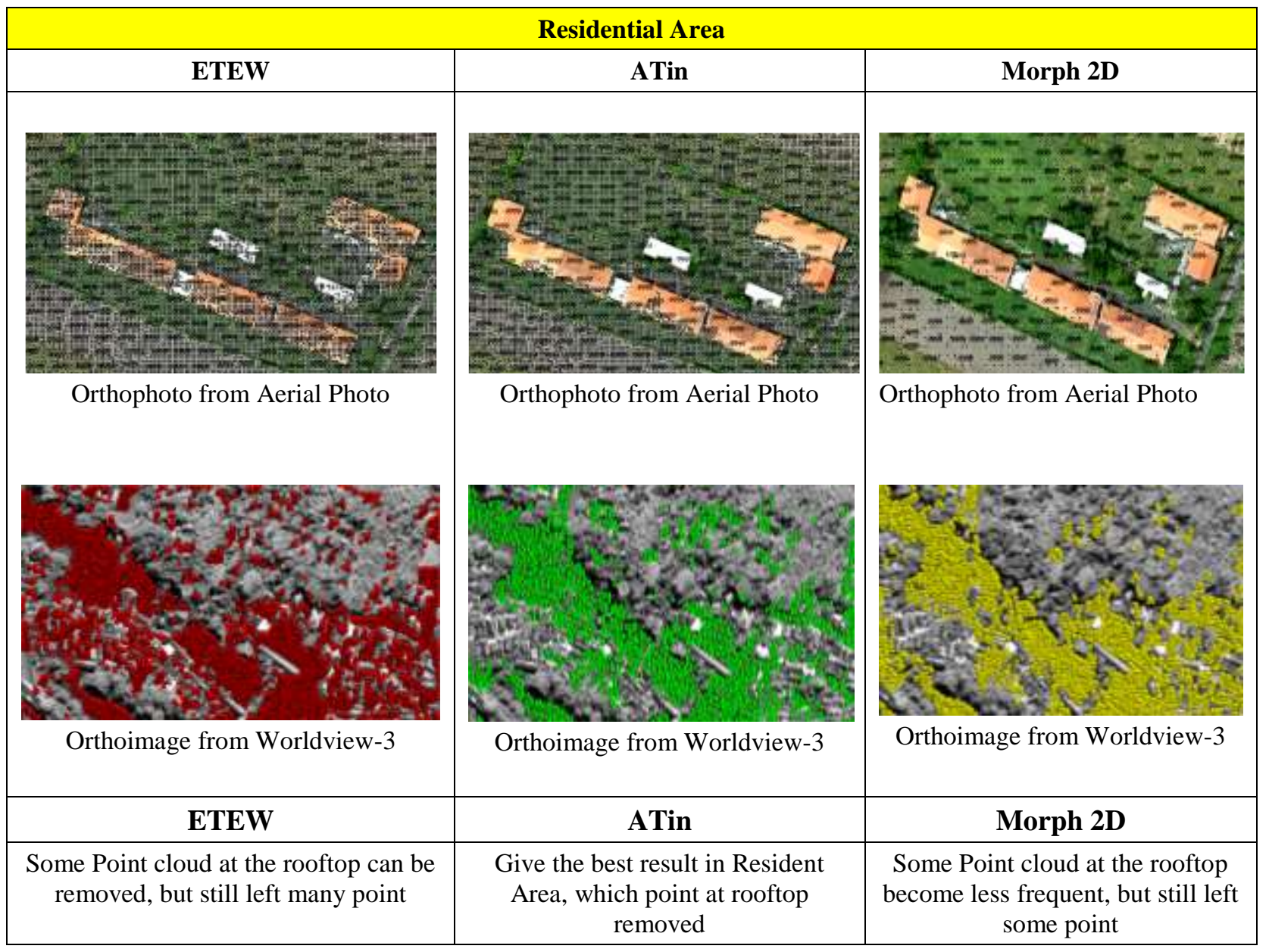

Table 2 explains the visualization of filtering results by using 3 algorithm methods, including ETEW, ATIN, Morph 2D method in the residential area. The ETEW method for residential areas, there are still some clouds on the roof but can be removed, on this method it still leaves a lot of dots. The ATIN method for the residential area is a method with the best results that point on the roof can be removed. The 2D Morph method for residential areas there are still some cloud spots on the roof but lacking many still leave some point.

Table 3 explains the visualization of filtering results by using 3 algorithm methods, including ETEW, ATIN, Morph 2D method in the vegetation area. On the ETEW method for the vegetation area for the tree object can be deleted still leave some dots. On the ATIN method for vegetation is a method with the best results with dots on the tree object can be removed. In the method of the 2D Morph for vegetation, there are still some points on tree objects but less numerous. 
Table 3. Result from DSM Filtering in Vegetation Area

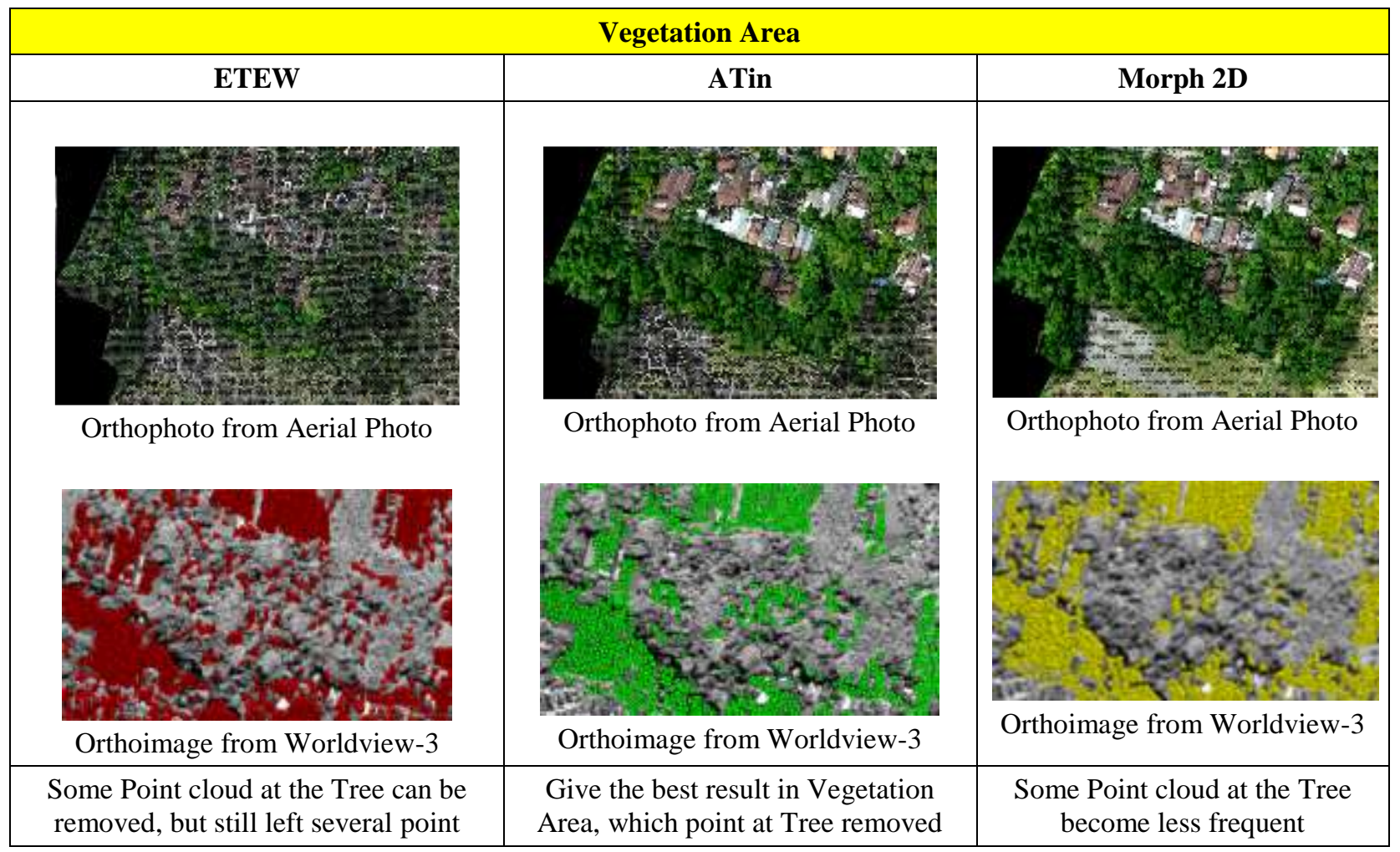

The comparison between three algorithm shows that Adaptive TIN give the best result. The ATin method provides the best results because the number of non-terrain point clouds can be removed rather dominant. ATin algorithm also works both in residential and vegetation area. Adaptive TIN algorithm has the ability to handle surfaces with discontinuities deviation. Unfortunately, there are still some non-terrain point exist. This is because the parameter setting cannot working for removing all kind of objects in the earth surface. In this case, the process should be repeated until all the non-terrain point has removed. There is no guarantee that parameter algorithm can working for all landscape area also.

\subsection{Final DTM}

Processing DSM filtering produce bare-earth point that close to terrain point. While the non-terrain point removed. This condition make missing point in some areas. The missing point should be filled by interpolation. Figure 2 and 3 shows the comparison between DSM and DTM derived from filtering procedures in vegetation areas. It can be shown that there is still some non-terrain point at dense vegetation and residential area. This non-terrain point makes contour like bull eyes shape. The non-terrain point remaining can be detected as spike or pit in elevation value. It is need to repeat filtering procedures for removing remaining non-terrain point.

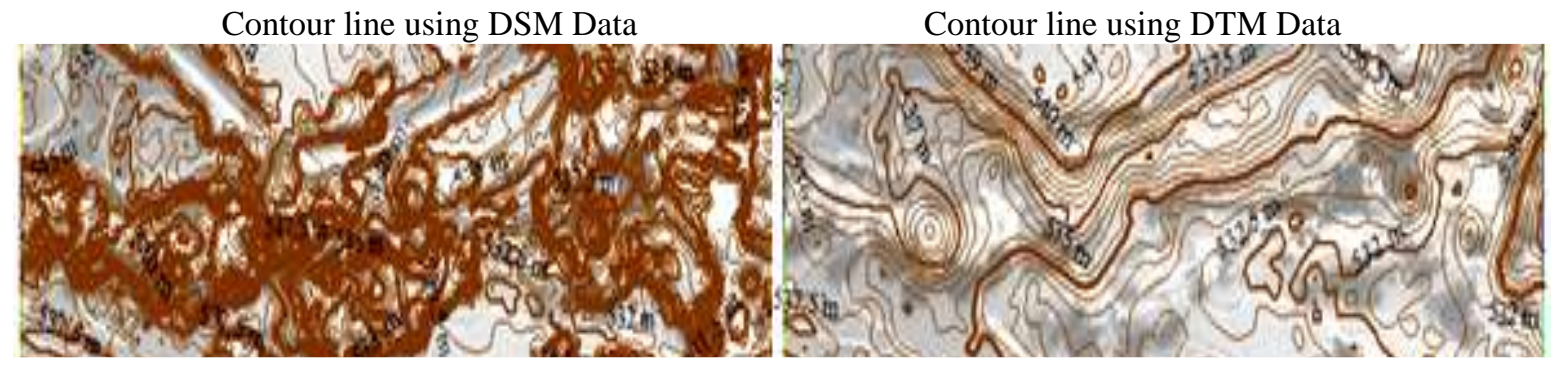

Figure 2. Contour line at residential area 

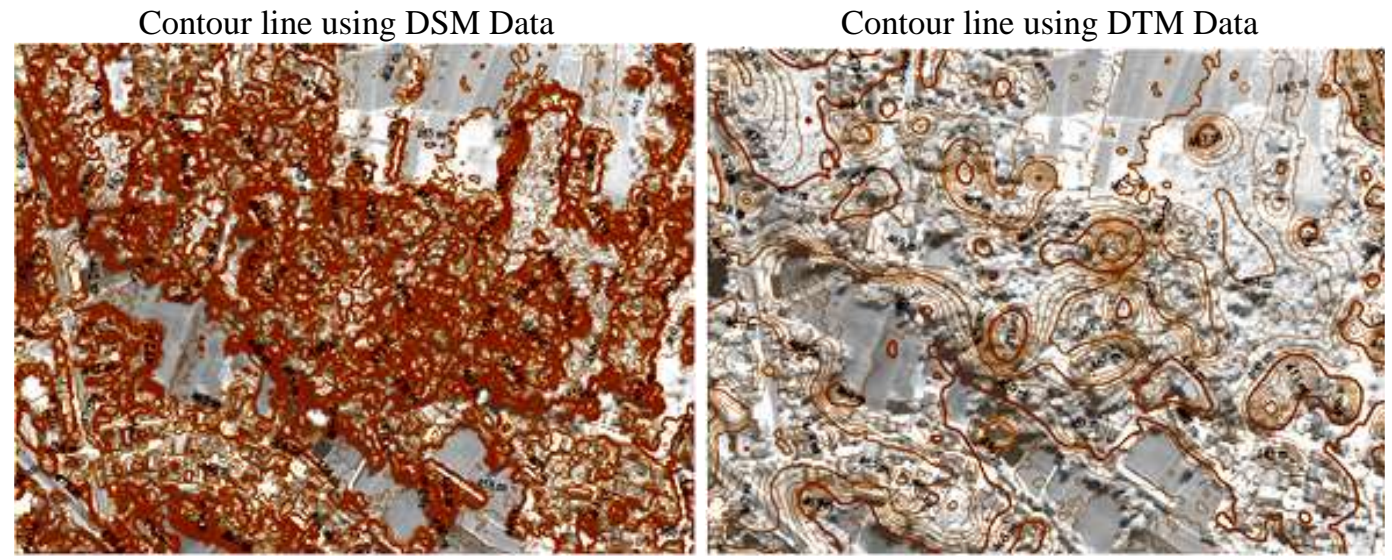

Figure 3. Contour line at vegetation area

\section{CONCLUSION}

This paper shows some algorithm for DSM filtering to remove a non-terrain point cloud. The use of different algorithms produces different remaining terrain points. The Adaptive TIN algorithm give the best result for DSM filtering procedure followed by ETEW and Morph 2D algorithm. The filtering algorithm with ATin and ETEW can working in residential and vegetation at any landscape area. While Morph 2D algorithm can be used in flat area. There are still some nonterrain point remaining after DSM filtering, because the setting parameter algorithm cannot working for varies object at the surface of the earth. In this case, the iteratively filtering process should be made. Furthermore, an interpolation process should be made for filling the missing non-terrain point.

\section{REFERENCES}

[1] Cheng, Ph., Chaapel, C., Using WorldView-1 stereo data with or without ground control points. GEOinformatics, 11 (7), pp. 34-39 (2008)

[2] Hu F., X.M. Gao, G.Y.Li M.Li, DEM Extraction From Worldview-3 Stereo-Images and Accuracy Evaluation, The International Archives of the Photogrammetry, Remote Sensing and Spatial Information Sciences, Volume XLI-B1, 2016 XXIII ISPRS Congress, 12-19 July 2016, Prague, Czech Republic p 327-332 (2016)

[3] Capaldo, P., Crespi, M., Fratarcangeli, F., Nascetti, A., Pieralice, F., DSM generation from high resolution imagery: applications with WorldView-1 and GeoEye-1. European Journal of Remote Sensing, 44(1), pp.41-53 (2012)

[4] Zhang, K., Chen, S., Whitman, D., Shyu, M., Yan, J. and Zhang, C., A progressive morphological filter for removing non-ground measurements from airborne LIDAR data. IEEE Transactions on Geoscience and Remote Sensing, 41, 872-882 (2003)

[5] Zhang, K. and Whitman, D., Comparison of three algorithms for filtering airborne LIDAR data. Photogrammetric Engineering and Remote Sensing, 71, 313-324 (2005)

[6] Axelsson, P., DEM generation from laser scanner data using adaptive tin models. International Archives of Photogrammetry and Remote Sensing, XXXIII, Part B3, 85-92 (2000)

[7] Sithole, G. and Vosselman, G., Experimental comparison of filter algorithms for bare-Earth extraction from airborne laser scanning point clouds. ISPRS Journal of Photogrammetry and Remote Sensing, 59, 85-101 (2004)

[8] Axelsson, P. DEM Generation From Laser Scanner Data Using Adaptive TIN Model. International Archives of Photogrammetry and Remote Sensing. Vol. 33. Amsterdam. (2000)

[9] Sulaiman N S, Majid Z, and Setan H., DTM Generation From Lidar Data By Using Different Filters In Open Source Software. Geoinformation Science Journal, 10(2), 89-109 (2010) 